\title{
Levantamento epidemiológico e fatores associados à coinfecção tuberculose/HIV no Brasil
}

\author{
Epidemiological survey and factors associated with tuberculosis / HIV co-infection in Brazil \\ Encuesta epidemiológica y factores asociados a la coinfección tuberculosis / VIH en Brasil \\ Letícia Fonseca Macedo ${ }^{1 *}$, Thalita da Rocha Bastos¹, João Victor Salvador Deprá1, Leonardo \\ Pereira Puget Feio², Thaisy Luane Gomes Pereira Braga' ${ }^{1}$, Andréa Luzia Vaz Paes ${ }^{1,2}$.
}

\section{RESUMO}

Objetivo: Realizar levantamento sobre os aspectos epidemiológicos e sociodemográficos de casos de coinfecção tuberculose/Vírus da Imunodeficiência Humana (HIV), e comparar o encontrado com os dados presentes na base Sistema de Informações de Agravos de Notificação (SINAN) acerca do número de casos dessa coinfecção no Brasil entre 2016 a 2019. Métodos: Trata-se de uma revisão integrativa da literatura, realizada por meio de coleta de dados a partir das bases de dados Scientific Electronic Library Online (SCIELO), Literatura Internacional em Ciências da Saúde (MEDLINE) e Literatura Latino-Americana em Ciências da Saúde (LILACS), com dados dos últimos cinco anos (2016-2020), e SINAN. Resultados: Evidenciou-se que há um predomínio da coinfecção TB/HIV em alguns grupos, como: homens, classe economicamente ativa entre 15 a 60 anos, raça parda e/ou negra, baixo nível socioeconômico, usuários de drogas ilícitas e etilistas. Conclusão: Infere-se que houve uma concordância entre os dados dos estudos analisados e os achados na plataforma do Sistema Único de Saúde (SINAN). Dessa forma, entende-se que as variáveis pesquisadas são importantes determinantes sociais na saúde, uma vez que auxiliam no controle e na vigilância das doenças infeciosas, como Tuberculose e HIV.

Palavras-chave: Tuberculose, Coinfecção pelo HIV, Epidemiologia.

\begin{abstract}
Objective: Conduct a survey on the epidemiological and sociodemographic aspects of cases of tuberculosis/Human Immunodeficiency Virus (HIV) coinfection, and compare what was found with the data present in the Notifiable Diseases Information System (SINAN) database on the number of cases of this coinfection in Brazil between 2016 to 2019. Methods: It is an integrative literature review, carried out through data collection from the databases Scientific Electronic Library Online (SCIELO), International Literature in Health Sciences (MEDLINE) and Latin American Literature in Health Sciences (LILACS), with data from the last five years (2016-2020), and SINAN. Results: It was evident that there is a predominance of TB / HIV coinfection in some groups, such as: men, economically active class between 15 to 60 years old, mixed race and / or black, low socioeconomic level, users of illicit drugs and alcohol users. Conclusion: It is inferred that there was an agreement between the data of the studies analyzed and the findings on the Unified Health System (SINAN) platform. Thus, it is understood that the researched variables are important social determinants in health, since they help in the control and surveillance of infectious diseases, such as Tuberculosis and HIV.
\end{abstract}

Keywords: Tuberculosis, HIV co-infection, Epidemiology.

\footnotetext{
1 Universidade do Estado do Pará (UEPA), Belém - PA.

${ }^{*}$ E-mail: leticiafm@live.com

${ }^{2}$ Centro Universitário do Estado Pará (CESUPA), Belém - PA.
}

SUBMETIDO EM: 9/2020

ACEITO EM: 10/2020

PUBLICADO EM: 1/2021 


\section{RESUMEN}

Objetivo: Realizar una encuesta de los aspectos epidemiológicos y sociodemográficos de los casos de coinfección tuberculosis / Virus de Inmunodeficiencia Humana (VIH) y comparar lo encontrado con los datos presentes en la base de datos del Sistema de Información de Enfermedades de Notificación (SINAN) sobre el número de casos de esta coinfección en Brasil entre 2016 y 2019. Métodos: Se trata de una revisión integradora de la literatura, realizada mediante recolección de datos en las bases de datos de la Biblioteca Electrónica Científica en línea (Scielo), Literatura Internacional en Ciencias de la Salud (MEDLINE) y Literatura Latinoamericana en Ciencias de la Salud (LILACS), con datos de los últimos cinco años (2016-2020), y SINAN. Resultados: Se evidenció que existe predominio de la coinfección TB / VIH en algunos grupos, tales como: hombres, clase económicamente activa entre 15 a 60 años, morenos y / o negros, nivel socioeconómico bajo, usuarios de drogas ilícitas. usuarios de drogas y alcohol. Conclusión: Se infiere que hubo concordancia entre los datos de los estudios de gasto y los hallazgos de la plataforma del Sistema Único de Salud (SINAN). Así, se entiende que las variables investigadas son importantes determinantes sociales en salud, ya que ayudan en el control y vigilancia de enfermedades infecciosas, como la tuberculosis y el VIH.

Palabras clave: Tuberculosis, Coinfección VIH, Epidemiología.

\section{INTRODUÇÃO}

A tuberculose (TB) é uma doença infecciosa causada pelo Mycobacterium tuberculosis (MTB) (LIMA SS, et al., 2017). A transmissão da doença se dá por meio da eliminação dos bacilos pelo portador, e o grau de infectividade é dependente da condição imunológica da pessoa exposta aos agentes.

Apesar da existência de medidas terapêuticas e de prevenção bem desenvolvidas, a TB continua a ser uma doença de prevalência significativa nos países subdesenvolvidos, sendo a principal causa de morte por um único agente de doença infecciosa em âmbito global (MACNEIL A, et al., 2019).

Dessa maneira, vale a pena ressaltar que o Brasil ocupa o $20^{\circ}$ lugar dentre os países com maior número de casos de tuberculose em proporção global, com cerca de 46 casos por 100.000 habitantes/anos. De outro modo, atualmente já está bem comprovada a maior incidência da Tuberculose em populações em situações socioeconomicamente desfavoráveis, com relação acentuada entre a pobreza, a exclusão social e o agravo em saúde (LIMA SS, et al., 2017).

De outra forma, além de continuar sendo considerada endêmica entre a população em condição desfavorável, com o surgimento da AIDS/HIV, a tuberculose passou a representar uma ameaça maior à saúde, uma vez que a coinfecção tuberculose-HIV é responsável por alterar a epidemiologia, evolução e prognóstico da doença. Além disso, estudos apontam que a tuberculose está entre as três doenças oportunistas de maior frequência em pessoas com HIV. Dessa forma, a infecção por tuberculose é responsável por cerca de $20 \%$ a $40 \%$ das mortes em pessoas HIV positivas (BLACK TLP, et al., 2016).

O Vírus da imunodeficiência humana (HIV) pertence à classe dos retrovírus e é responsável por causar a Síndrome da imunodeficiência adquirida (AIDS). Sendo transmitido principalmente por relação sexual desprotegida, é hoje considerado um dos maiores problemas de saúde pública no Brasil (GALVÃO JMV, et al., 2019)

Pessoas com HIV são cerca de 30 vezes mais propensas a adquirir tuberculose, infecção oportunista que atua reduzindo a contagem de linfócitos TCD4 e aumentando a carga viral nessas pessoas. Esse fenômeno é conhecido como transativação heteróloga e é causado por ação direta do Mycobacterium tuberculosis (OLIVEIRA LB, 2018; OLIVEIRA EH, 2020).

Além disso, o HIV aumenta a chance de ocorrer a transição da forma latente da tuberculose para a sua forma ativa. Uma das formas de proteção imune contra a progressão da doença é por meio da apoptose. Porém, em indivíduos com coinfecção, a apoptose dos macrófagos alveolares está comprometida, ocorrendo 
então persistência do MTB no interior das células e piora do quadro evolutivo da doença (COINCEIÇÃO EL, et al., 2016).

Dessa forma, tem-se a coinfecção tuberculose-HIV como agente propiciador do aumento da taxa de morbidade e mortalidade associada à tuberculose. Em pessoas imunocompetentes, o risco de a infecção pelo vírus da tuberculose evoluir para a forma ativa da doença é de $10 \%$ em toda a vida, porém em pessoas imunodeprimidas, essa porcentagem de progressão sobe para $10 \%$ ao ano (MIRANDA LO, et al., 2017). Além disso, a taxa de mortalidade em coinfectados é de $19 \%$, enquanto a de pessoas sem HIV que adquirem tuberculose é de 2,4\% (PEREIRA LFB, et al., 2018).

Sendo assim, tendo em vista a importância da epidemiologia para o planejamento das ações em saúde e de medidas de controle das doenças, este estudo tem como objetivo realizar um levantamento acerca dos atuais conhecimentos na literatura científica sobre os aspectos epidemiológicos e sociodemográficos de casos de coinfecção tuberculose/HIV, nos últimos cinco anos e comparar o encontrado na literatura com os dados presentes na base Sistema de Informações de Agravos de Notificação (SINAN) acerca do número de casos dessa coinfecção no Brasil entre 2016 a 2019.

\section{MÉTODOS}

Trata-se de um estudo de revisão integrativa da literatura, realizada por meio de uma busca sistemática nas bases de dados Scientific Electronic Library Online (SCIELO), Literatura Internacional em Ciências da Saúde (MEDLINE/Pubmed) e Literatura Latino-Americana em Ciências da Saúde (LILACS), sendo contabilizados para a amostra materiais com data de publicação dos últimos cinco anos, abrangendo os anos de 2016 a 2020. Bem como, foi feito um levantamento acerca do número de casos de coinfecção por Tuberculose e HIV/AIDS no período de 2016 a 2019 com base nos dados do SINAN.

Foram utilizados os seguintes descritores como estratégia de busca: "Tuberculose"; "Coinfecção"; "HIV"; "Epidemiologia". Desses foram analisadas as seguintes variáveis: sexo, faixa etária, escolaridade, cor/raça, nível socioeconômico, uso de drogas ilícitas e alcoolismo.

Quanto às variáveis utilizadas para pesquisa na base de dados do SINAN, foram consideradas: ano de ocorrência, região, faixa etária, sexo, situação de encerramento, escolaridade, cor/raça e uso de drogas ilícitas. Além disso, levou-se em consideração o número de doentes com TB que foram testados para HIV, como recomenda o Ministério da Saúde.

Como critérios de inclusão foram selecionados artigos em português, em espanhol e em inglês que apresentaram a descrição do perfil epidemiológico dos coinfectados com TB/HIV no cenário brasileiro. Ainda, foram descartados artigos que não abordassem pelo menos quatro das variáveis analisadas.

A presente revisão de artigos foi realizada em duas etapas. Inicialmente, os artigos encontrados nas bases de dados foram avaliados quanto ao título, leitura do resumo e palavras-chave, sendo elegidos os que mencionavam o perfil epidemiológico dos casos de tuberculose coinfecção HIV.

Restando, assim, 28 trabalhos para serem lidos integralmente buscando aqueles que melhor se adequaram aos critérios estabelecidos nessa revisão. A análise dos resultados foi realizada com base na amostragem do número total de casos e a partir deste foi calculada a frequência relativa de cada variável em relação ao $n$ amostral de artigos selecionados para análise.

\section{RESULTADOS}

Foram encontrados 718 estudos, sendo 15 LILACS, 9 Scielo, 694 Medline/Pubmed. Respeitando os critérios dessa revisão integrativa da literatura, 28 foram identificados em uma pré-seleção, 15 artigos foram considerados elegíveis por preencherem os critérios de inclusão (Quadro 1). 
Quadro 1 - Sumário dos estudos analisados.

\begin{tabular}{|c|c|c|c|c|c|c|}
\hline Citação & Sexo & $\begin{array}{c}\text { Faixa } \\
\text { Etária(anos) }\end{array}$ & Escolaridade & Cor/raça & $\begin{array}{c}\text { Nível } \\
\text { Socioeconômico }\end{array}$ & $\begin{array}{l}\text { Uso de } \\
\text { Drogas/ } \\
\text { Alcoolismo }\end{array}$ \\
\hline $\begin{array}{c}\text { BASTOS SH, et al. Perfil Sociodemográfico e de saúde da } \\
\text { coinfecção tuberculose/HIV no Brasil: revisão sistemática. Rev } \\
\text { Bras Enferm. 2019;72(5):1458-65. }\end{array}$ & Masculino & $15-60$ & $\begin{array}{l}\text { Ens. Fund. } \\
\text { Incompleto }\end{array}$ & $\begin{array}{l}\text { Parda e } \\
\text { preta }\end{array}$ & Baixa renda & Etilismo \\
\hline $\begin{array}{l}\text { MANN D, et al. Cutaneous tuberculosis and HIV infection at a } \\
\text { referral centre in Rio de Janeiro, Brazil. Mem Inst Oswaldo Cruz. } \\
2018 ; 113(9): 1-6 .\end{array}$ & Masculino & $24-52$ & $\begin{array}{l}\text { Ens. Fund. } \\
\text { Incompleto }\end{array}$ & $\begin{array}{c}\text { Não } \\
\text { branca }\end{array}$ & - & $\begin{array}{l}\text { Uso } \\
\text { excessivo } \\
\text { de álcool e } \\
\text { de drogas } \\
\text { ilícitas }\end{array}$ \\
\hline $\begin{array}{l}\text { QUEIROZ CA, et al. Tuberculose associada à AIDS: uma análise } \\
\text { da prevalência de coinfecção. Ciência et Praxis. 2018;11(21):65- } \\
\qquad 70 \text {. }\end{array}$ & Masculino & $31-40$ & $\begin{array}{l}\text { Ens. Fund. } \\
\text { Incompleto }\end{array}$ & - & Baixa renda & Etilismo \\
\hline $\begin{array}{l}\text { ROSSETTO M, et al. Coinfecção tuberculose/HIV/aids em Porto } \\
\text { Alegre, RS -invisibilidade e silenciamento dos grupos mais } \\
\text { afetados. Rev. Gaúcha Enferm. 2019;40(e20180033):1-9. }\end{array}$ & $\begin{array}{l}\text { Masculino } \\
/ \\
\text { Feminino }\end{array}$ & Média de 38 & $\begin{array}{c}\text { Baixa } \\
\text { escolaridade }\end{array}$ & Preta & Baixa renda & $\begin{array}{l}\text { Uso de } \\
\text { drogas e } \\
\text { álcool }\end{array}$ \\
\hline $\begin{array}{l}\text { SIQUEIRA-FILHA NT, et al. The economic burden of HIV and } \\
\text { TB/HIV co-infection in a middle-income country: a costing analysis } \\
\text { alongside a pragmatic clinical trial in Brazil. Sexually Transmitted } \\
\text { Infections. } 2018 ; 94(6): 463-469 .\end{array}$ & Masculino & $18-39$ & $\begin{array}{l}\text { Ens. Fund. } \\
\text { Incompleto }\end{array}$ & - & $\begin{array}{l}\text { Menos que } 1 \\
\text { salário mínimo }\end{array}$ & $\begin{array}{l}\text { Álcool e } \\
\text { drogas } \\
\text { ilícitas }\end{array}$ \\
\hline $\begin{array}{c}\text { SILVA BEB, et al. Prevalence of coinfections in women living with } \\
\text { human immunodeficiency virus in Northeast Brazil. Rev Soc Bras } \\
\text { Med Trop. 2020;53(e20190282):1-6. }\end{array}$ & Feminino & $26-49$ & $\begin{array}{l}\text { Ens. Fund. } \\
\text { Incompleto }\end{array}$ & Parda & $\begin{array}{l}\text { Até um a dois } \\
\text { salários mínimos }\end{array}$ & $\begin{array}{l}\text { Uso de } \\
\text { drogas } \\
\text { ilícitas }\end{array}$ \\
\hline $\begin{array}{c}\text { GONÇALVES HF. Perfil clínico e epidemiológico e prevalência de } \\
\text { coinfecção de tuberculose-hiv no estado de Pernambuco, Brasil. } \\
\text { Dissertação (Graduação) - Centro Acadêmico de Vitória de Santo } \\
\text { Antão. Universidade Federal de Pernambuco, Pernambuco; 2018, } \\
\text { 39p. }\end{array}$ & Masculino & $20-40$ & $\begin{array}{l}\text { Ens. Fund. } \\
\text { Incompleto }\end{array}$ & Parda & - & - \\
\hline
\end{tabular}




\begin{tabular}{|c|c|c|c|c|c|c|}
\hline Citação & Sexo & $\begin{array}{c}\text { Faixa } \\
\text { Etária(anos) }\end{array}$ & Escolaridade & Cor/raça & $\begin{array}{l}\text { Nível } \\
\text { Socioeconômico }\end{array}$ & $\begin{array}{l}\text { Uso de } \\
\text { Drogas/ } \\
\text { Alcoolismo }\end{array}$ \\
\hline $\begin{array}{c}\text { ROSSETTO M, et al, Teixeira LB. Perfil epidemiológico de los } \\
\text { casos de tuberculosis con coinfección VIH en Porto Alegre. Rev } \\
\text { Bras Enferm. 2019;72(5):1211-1218. }\end{array}$ & Masculino & $\begin{array}{l}\text { Média de } \\
\text { idade de } 38 \\
\quad \pm 9,91\end{array}$ & $\begin{array}{l}\text { Ens. Fund. } \\
\text { Incompleto }\end{array}$ & Branca & - & Etilismo \\
\hline $\begin{array}{l}\text { MARQUES CC, et al. Casos de tuberculose coinfectados por HIV } \\
\text { em um estado do nordeste brasileiro. Rev Enferm Act. } \\
\text { 2019;36:62-76. }\end{array}$ & Masculino & $20-39$ & $\begin{array}{l}\text { Ens. Fund. } \\
\text { Incompleto }\end{array}$ & Parda & - & $\begin{array}{l}\text { Drogas } \\
\text { ilícitas/ } \\
\text { tabagismo }\end{array}$ \\
\hline $\begin{array}{l}\text { CASTRIGHINI CC, et al. Prevalência e aspectos epidemiológicos } \\
\text { da coinfecção HIV/tuberculose. Rev enferm UERJ. 2017;25:1-6. }\end{array}$ & Masculino & 35 a 44 & $\begin{array}{l}\text { Ens. Fund. } \\
\text { Incompleto }\end{array}$ & Branca & - & - \\
\hline $\begin{array}{l}\text { TEIXEIRA F, et al. Human Immunodeficiency Virus and } \\
\text { Tuberculosis Coinfection in a Tertiary Hospital in Southern Brazil: } \\
\text { Clinical Profile and Outcomes. Microbiol Insights. 2018;11:1-8. }\end{array}$ & Masculino & $25-37$ & $\begin{array}{l}\text { Ens. Médio } \\
\text { Incompleto }\end{array}$ & - & - & $\begin{array}{l}\text { Uso de } \\
\text { drogas } \\
\text { injetáveis }\end{array}$ \\
\hline $\begin{array}{l}\text { LIMA MD, et al. Mortalidade relacionada à coinfecção tuberculose } \\
\text { e HIV/AIDS no Brasil, 2000-2011: padrões epidemiológicos e } \\
\text { tendências temporais. Cad. Saúde Pública. 2016;32(10):1-11. }\end{array}$ & Masculino & $30-39$ & $\begin{array}{l}\text { Ens. Fund. } \\
\text { Incompleto }\end{array}$ & Preta & - & - \\
\hline $\begin{array}{l}\text { PRADO TN, et al. Clinical and epidemiological characteristics } \\
\text { associated with unfavorable tuberculosis treatment outcomes in } \\
\text { TB-HIV co-infected patients in Brazil: a hierarchical polytomous } \\
\text { analysis. Braz J Infect Dis. } 2017 ; 21(2): 162-170 .\end{array}$ & Masculino & $20-59$ & $\begin{array}{l}\text { Ens. Fund. } \\
\text { Incompleto }\end{array}$ & - & - & Etilismo \\
\hline $\begin{array}{l}\text { FERREIRA DP, et al. Prevalência da Coinfecção Hiv/Tb em } \\
\text { Pacientes de um Hospital de Referência na Cidade do Rio De } \\
\text { Janeiro. J. Rev Fundam. Care. Online.2019; 11: 358-362 }\end{array}$ & Masculino & $\begin{array}{l}\text { Média de } \\
\quad 37,5\end{array}$ & Ens. Superior & Preta & - & - \\
\hline $\begin{array}{l}\text { MAGNO ES, et al. Fatores associados à coinfecção tuberculose e } \\
\text { HIV: o que apontam os dados de notificação do Estado do } \\
\text { Amazonas, Brasil, 2001-2012. Cad. Saúde Pública. 2017; 35(5): } \\
\text { 1-11. }\end{array}$ & Maculino & $25-40$ & - & Parda & - & $\begin{array}{l}\text { Uso de } \\
\text { drogas }\end{array}$ \\
\hline
\end{tabular}

Fonte: Macedo LF, et al., 2020 
Dos estudos encontrados, a maioria $(86,87 \%)$ apresentou o sexo masculino como o mais afetado pela coinfecção TB/HIV, com exceção de um, os quais constataram as mulheres como mais prevalentes, e um realizado em Porto alegre, no qual ambos os sexos foram igualmente atingidos. Já em relação à faixa etária com maior número de casos, todos os artigos mostraram ser a classe economicamente ativa, variando de 15 a 60 anos. A raça mais prevalente foi a parda e a preta $(60,00 \%)$, e a com menor prevalência foi a branca (13,33\%). Vale destacar ainda que $26,67 \%$ não apresentavam dados sobre cor/raça.

A análise quanto ao nível socioeconômico demonstrou prevalência de baixo nível econômico em todos os artigos analisados (33,33\%). Porém, dez (66,67\%) artigos não continham informações quanto à essa variável. Ainda $46,67 \%$ dos estudos identificaram relação entre o alcoolismo e a coinfecção TB/ HIV. 46,67\% também relacionou a coinfecção com o uso de drogas ilícitas. Vale destacar que quatro (26,67\%) estudos não apresentaram dados quanto ao uso de drogas ou álcool. Em relação à escolaridade, houve prevalência de Ensino Fundamental Incompleto (80,00\%), seguido do Ensino Médio Incompleto e Ensino Superior que corresponderam (13,33\%).

De acordo com a pesquisa no (SINAN) do Brasil, foram notificados 38.000 casos confirmados de Tuberculose com coinfecção por HIV entre os anos de 2016 a 2019 no país. Na análise quanto aos anos, 2017 foi o que apresentou o maior número de casos, com 9.818 (25,84\%), e o ano de 2019 foi o que apresentou o menor, com 8.972 (23,61\%). Já 2016 e 2018 contaram com 9.501 (25,00\%) e 9.709 (25,55\%) casos, respectivamente. A região Sudeste apresentou o maior número de casos ( $n=15.199 ; 40,00 \%$ ), seguido pela região Nordeste $(n=8.859 ; 23,31 \%)$, a região Centro-oeste notificou o menor número $(n=1.720 ; 4,53 \%)$. Sul e Norte contaram com $7.763(20,43 \%)$ e 4.459 (11,73\%) casos, respectivamente.

Quanto à faixa etária mais acometida foi em adultos jovens entre 20 e 39 anos, com 20.544 (54,06\%), seguidos pelos indivíduos de 40 a 59 anos ( $n=14.727 ; 38,76 \%)$, indivíduo entre 0 a 19 anos apresentaram 948 casos (2,49\%) e entre 60 anos ou mais, com $1.774(4,67 \%)$, sendo que $7(0,02 \%)$ não apresentavam informações sobre essa variável. Em relação ao sexo, o masculino apresentou maior número, com 27.309 $(71,86 \%)$, seguido do feminino com $10.688(28,12 \%)$, sendo que $3(0,02 \%)$ não apresentavam informações dessa variável. A raça parda foi a mais acometida, com 17.830 (46,92\%) casos, seguida pela branca, com $11.418(30,05 \%)$ e preta, com 5.720 (15,05\%). A raça amarela e os indígenas contaram com $178(0,47 \%)$ e $118(0,31 \%)$ casos respectivamente. $2.736(7,20 \%)$ casos foram ignorados quanto a essa variável.

No tocante à escolaridade, a maior parte dos casos ocorreu em pessoas com ensino fundamental incompleto, com $12.617(33,20 \%)$ casos, já o ensino superior incompleto obteve o menor número, com 1.021 (2,69\%) (Gráfico 1).

Gráfico 1 - Número e porcentagem dos níveis de escolaridade analisados no SINAN.

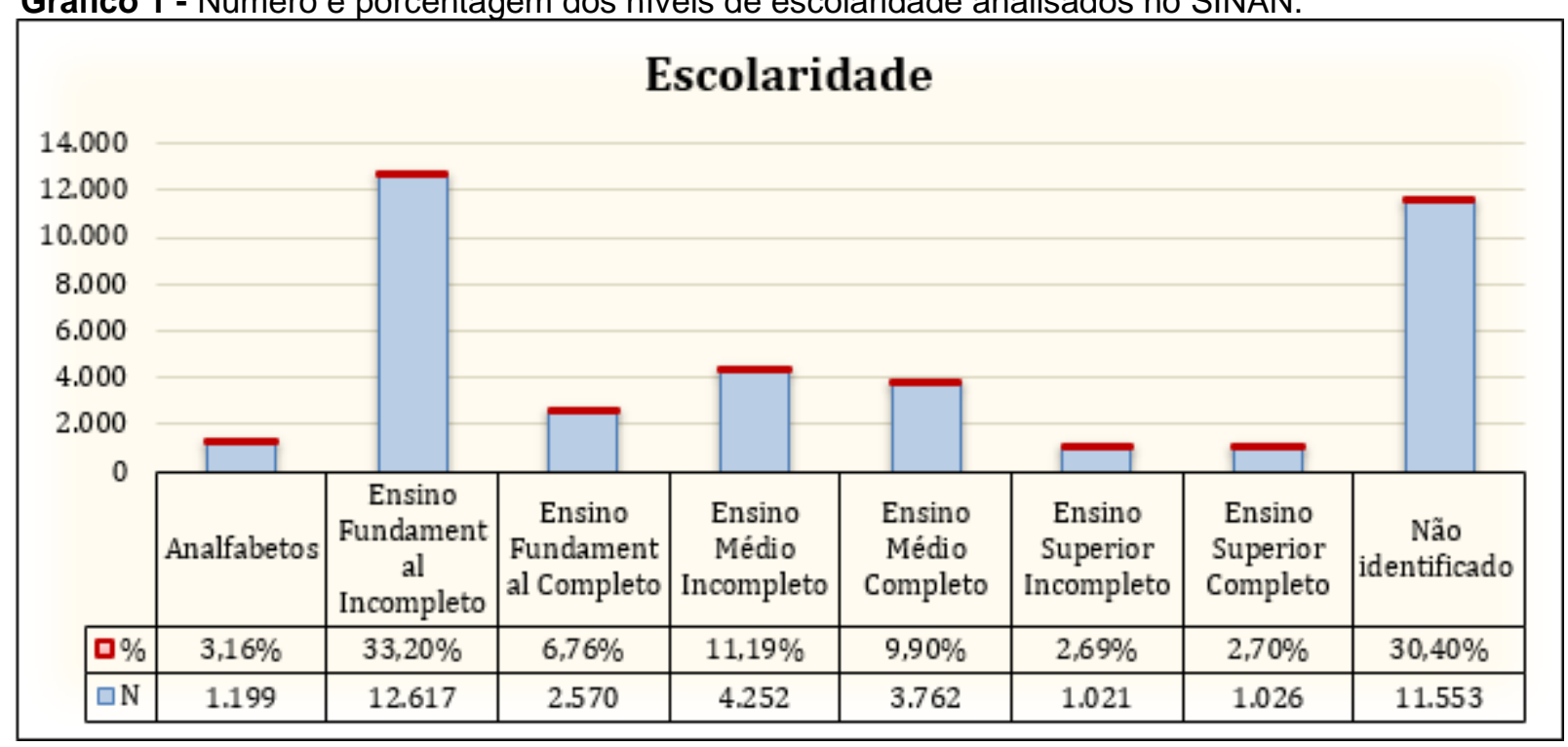

Fonte: MACEDO LF, et al., 2020. 
No que se refere à situação de encerramento, $37,6 \%(n=14.308)$ conseguiram se curar da doença, $15,2 \%$ (5.780) foram óbitos por outras causas, sendo que 2,8\% ( $n=1.049)$ evoluíram ao óbito por tuberculose, 6.186 $(16,27 \%)$ abandonaram o tratamento e 15,6\% (n=5.954) não apresentaram informações sobre essa variável. Ademais, dos 38.000 casos, $24,96 \%$ ( $n=9.485)$ ocorreram em usuários de drogas ilícitas e 3.243 casos foram ignorados quanto à essa variável. Em relação a testagem para HIV dos doentes com TB, como recomenda o Ministério da Saúde, do total de 361.639 casos de tuberculose, 59.962 (16,58\%) doentes não foram testados para o HIV.

\section{DISCUSSÃO}

O presente estudo buscou analisar o perfil epidemiológico e os fatores associados a coinfecção TB/HIV, esperando-se que haja um melhor entendimento da realidade dessa comorbidade nos dias atuais. Além disso, ao traçar um perfil epidemiológico, pode-se lidar de forma mais eficaz com os grupos de risco, justificando traçar estratégias mais eficazes para suavizar os impactos dessa coinfecção no país. Por meio da análise dos resultados encontrados nesta revisão, verificou-se que, dentre os artigos que abordavam perfil sociodemográfico, a maior parcela de acometimento pela coinfecção TB/HIV é masculina ( $80 \% ; n=12$ artigos). No entanto, dois artigos demonstram as mulheres como a maioria acometida pelas doenças associadas e um artigo aponta distribuição equivalente de casos nos dois sexos.

Além disso, os dados obtidos do Sistema de Informação de Agravos de Notificação (SINAN) do Brasil confirmam essa realidade, demonstrando a prevalência masculina com cerca de $71,86 \%$ dos casos de TB/HIV, no período de 2016 a 2019. No entanto, um estudo transversal realizado em Sergipe, entre os anos de 2014 e 2017, analisou as mais frequentes coinfecções de mulheres brasileiras portadoras do vírus do HIV, e aponta, entre essas comorbidades, a tuberculose (SILVA WA, et al., 2017).

Com isso, pode-se inferir que, apesar da notável dominância masculina, a prevalência tanto do vírus do HIV como da tuberculose tem aumentado gradativamente no sexo feminino. Já o estudo de Oliveira HB, et al. (2019), o sexo masculino constituiu a grande maioria dos casos $(68,4 \%)$ e dos óbitos $(74,1 \%)$, tanto entre pacientes com coinfecção TB/HIV quanto entre pacientes com TB não-HIV.

Finalmente, não foram encontrados dados na literatura que explicam essa disparidade entre os sexos, contudo, pode-se relacionar essa questão a fatores biológicos, hábitos de vida e o fato de os homens historicamente buscarem tardiamente os sistemas de saúde (SANTOS MLSG, et al., 2009). Ademais, a respeito da faixa etária mais acometida, foi possível encontrar uma prevalência da idade economicamente ativa, variando de 15 a 54 anos.

$\mathrm{Na}$ África Subsaariana, uma das regiões mais gravemente afetadas pela epidemia HIV/AIDS, os adultos jovens, população de 15 a 49 anos, pertencem ao grupo mais afetado pela infecção pelo M. tuberculosis, bem como estão em maior risco de infecção pelo HIV, resultando em epidemias sobrepostas nessa faixa etária (BRUCHFELD J, et al., 2015).

A análise dos dados do SINAN mostrou uma convergência de informações com a literatura, revelando a prevalência da doença em adultos jovens entre 20 e 39 anos, com 54\% dos casos. Dessa forma, essa prevalência pode ser explicada pelo estilo de vida de adultos jovens, como a predisposição à poligamia, a não utilização de métodos contraceptivos de barreira e a utilização de drogas e álcool (SANTOS NETO M, 2012; HINO P, 2012).

Ademais, no estudo de Silva WA, et al. (2017), observou-se que a baixa incidência da doença (tuberculose) na faixa etária de 0 a 20 anos pode estar ligada às altas coberturas e à eficácia da vacinação com BCG, além da subnotificação, corroborando com o baixo número de casos nesse grupo quando comparado às demais faixas etárias pelos dados do SINAN $(2,49 \%)$.

Historicamente, a baixa escolaridade é um fator influente para o agravamento das doenças, tendo em vista que um baixo nível de instrução acarreta um baixo acesso à informação sobre os serviços de saúde e em desconhecimento acerca de sintomas e sinais clássicos de diversas comorbidades (SANTOS NETO M, et al., 2012). Além disso, estaticamente, são pessoas em estado de vulnerabilidade social, o que agrava ainda mais o quadro desse grupo. 
Diante da coinfecção TB/HIV, pode-se perceber a prevalência de pessoas com baixa escolaridade dentre os acometidos por essa condição, como mostra a pesquisa proposta por Santos AR, et al. (2020) e os dados do SINAN, que demonstram que $33,20 \%$ dos indivíduos acometidos possui ensino fundamental incompleto e apenas $2,70 \%$ dos acometidos com ensino superior completo.

Sendo assim, pode se perceber que essas doenças abrangem uma questão social particularmente importante no Brasil, tendo em vista a grande desigualdade social e as condições de vida precárias de uma grande parcela da população, o que facilita o crescente número de casos de coinfecção por TB/HIV no país.

A respeito da raça mais acometida, foi percebido que os indivíduos de pele parda e preta são os mais afetados pela coinfecção TB/HIV (sete dos nove artigos que tratavam dessa variável denotavam essa prevalência não branca). Essa disparidade de valores adquire diferenças ao se analisar os dados do SINAN, que denota a raça parda com $46,92 \%$ dos casos, seguida pela raça branca com $30,04 \%$ e pela preta, com apenas $15,05 \%$. No entanto, deve-se ressaltar que, em um estudo realizado em Porto Alegre, em 2019, foi executado um protocolo onde delimitou-se as regiões da cidade com maior expressividade de casos e de especificidades, tais como: sexo e raça.

Nesse cenário, traçou-se um perfil epidemiológico e foram identificadas condições que tiveram um importante papel no aumento da vulnerabilidade dos indivíduos, e, dentre estas, se encontrava o fator pele escura (ROSSETTO M, et al., 2019). Ainda, no estudo transversal de Escada ROS, et al. (2017), pacientes negros tiveram o dobro da taxa de mortalidade em comparação com pacientes brancos.

Ademais, o estudo transversal proposto por Gonçalves HF (2018) relaciona a maior prevalência das doenças em questão na população negra com a situação de vulnerabilidade social que esse grupo estatisticamente apresenta, sendo considerados, inclusive, menos privilegiados pelos sistemas de saúde e, concomitante a isso, denota que $67,3 \%$ dos casos avaliados eram das populações parda e negra de forma conjunta.

Portanto, é válido ressaltar que, por mais que ainda existam controvérsias, a maior taxa de acometimento pela coinfecção TB/HIV aparenta ser da população de pele parda e preta. A análise dos artigos permitiu identificar a baixa renda como um dos fatores epidemiológicos a serem considerados na coinfecção TB/HIV, sendo constatada em sete $(46,7 \%)$ dos artigos avaliados.

Com isso, pode-se inferir que essa comorbidade atua como uma problemática social, ultrapassando barreiras biológicas, de forma que os indivíduos mais acometidos são a parcela da população com menos acesso aos serviços de saúde e que, estaticamente, menos pratica o autocuidado.

Acerca disso, Vendramini SHF, et al. (2010) afirma que a vulnerabilidade dos indivíduos com baixo poder aquisitivo se dá, principalmente, pelo fato desses indivíduos não se enxergarem em risco, o que dificultaria a autopromoção de saúde. Até em países desenvolvidos, a coinfecção TB/HIV apresenta uma incidência maior na população vulnerável, como imigrantes e pessoas de baixa renda (ORCAU A, et al., 2011).

Além disso, a distribuição social das cidades também se relaciona com a renda de seus habitantes, fato que aumenta a segregação ao serem estabelecidas áreas de indisponibilidade de serviços de saúde, habitação e de reduzida atuação de políticas públicas, o que, mais uma vez, contribui para a maior prevalência da coinfecção TB/HIV entre esse grupo social (VENDRAMINI SHF, 2010; OLIVEIRA NF e GONÇALVES MJF, 2013).

No entanto, o considerável número de artigos da amostra dessa revisão $(53,3 \%)$ que não apresentam informações sobre essa variável limita uma análise epidemiológica mais específica. Por fim, a análise da literatura permitiu encontrar o uso de álcool, tabaco e outras drogas como fator predisponente à coinfecção TB/HIV. A análise dos dados do SINAN está de acordo com esse achado, como pode-se perceber ao verificar a prevalência de $24,96 \%$ do total dos casos como usuários de algum tipo de droga ilícita.

Além disso, o estudo proposto por Bastos SH, et al. (2019) aponta a porcentagem do uso de álcool entre os infectados de 50,8\%. Ainda, Sousa AG, et al. (2013) apontou as drogas ilícitas como um dos fatores para maior prevalência de TB/HIV no grupo de adultos jovens. 
Concomitante a isso, pode-se apontar o uso recreativo de álcool como um fator que prejudica a eficácia dos medicamentos e acaba ocasionando a interrupção da medicação, assim como o uso de drogas ilícitas atua como uma barreira para a adesão ao tratamento, afetando não só o paciente e sua relação com os intervalos entre os medicamentos e as drogas ingeridas, mas também a visão da equipe profissional sobre esse paciente, devido ao estereótipo difundido socialmente, o que impede um maior auxílio nas dificuldades apresentadas (SOUSA FILHO MP, et al., 2012).

Um importante ponto a ser acrescentando é um provável aumento de casos e número de mortes por tuberculose nos próximos quatro anos devido à pandemia global da COVID-19. Vários cenários foram analisados pelo estudo publicado no European Respiratory Journal, no entanto, no pior deles, o impacto da pandemia nos serviços de saúde, levando à sobrecarga desses devido ao alto número de casos de coronavírus, resultaria em severas reduções na disponibilidade e no acesso a esses serviços para diagnóstico e tratamento de casos de tuberculose (MCQUAID CF, et al., 2020).

Além disso, esse panorama é ainda mais desafiador em países subdesenvolvidos, onde a mortalidade por TB em jovens é altamente prevalente, ainda com fatores de risco associados como: tabagismo, abuso de álcool e substâncias, coinfecção pelo HIV, entre outros. Porém, o estudo não foi conclusivo se pacientes com tuberculose possuem um risco mais alto de evoluir para a forma mais grave da COVID-19 (TADOLINI M, et al., 2020).

Contudo, os pesquisadores sugerem que o distanciamento social poderia limitar a transmissão e a incidência do Mycobacterium tuberculoses, uma vez que esta bactéria causadora da tuberculose é transmitida através de gotículas no ar, semelhante ao coronavírus (MCQUAID CF, et al., 2020). Sendo assim, é imperativo que os órgãos de saúde garantam o acesso ao diagnóstico e ao tratamento da tuberculose, bem como atendimento e relatórios epidemiológicos contínuos da doença, a fim de minimizar e controlar o impacto da atual pandemia de coronavírus na tuberculose.

\section{CONCLUSÃO}

O desenvolvimento do presente estudo demonstrou uma análise que o gênero mais predominantemente acometido pela coinfecção TB/HIV é o sexo masculino, constituintes da população economicamente ativa de baixa renda, entre 20 e 39 anos e de cor parda. Além disso, permitiu-se observar a influência negativa de fatores como o alcoolismo, uso de drogas ilícitas e etilismo. No que diz respeito aos casos, notificou-se um total de 38.000 durante o período dos anos de 2016 a 2019 no Brasil. O ano de 2016 foi o mais prevalente, seguido por 2017, 2016 e o menos prevalente foi o ano de 2019. Quanto a região com maior número de casos notificados tem-se a região Sudeste. Em relação a escolaridade, pessoas com ensino fundamental incompleto foram maioria. É importante salientar que há uma escassez de pesquisas epidemiológicas recentes referentes ao assunto, principalmente no que se refere à condição de vida e aos hábitos dos indivíduos com TB/HIV. No que tange a situação de encerramento, mais de $1 / 3$ dos casos alcançaram cura da coinfecção. Por fim, a testagem para HIV dos doentes com TB mostrou-se presente em mais de $80 \%$ dos casos totais. Nesse sentido a atenção básica, devido a sua abrangência territorial, torna-se um elemento-chave para uma vigilância em saúde efetiva, que requer a compreensão dos determinantes sociais da saúde, o diagnóstico oportuno de agravos de relevância sanitária, principalmente na vigilância e no controle de doenças infectocontagiosas, como a coinfecção tuberculose/HIV.

\section{REFERÊNCIAS}

1. BASTOS SH, et al. Perfil Sociodemográfico e de saúde da coinfecção tuberculose/HIV no Brasil: revisão sistemática. Rev Bras Enferm. 2019;72(5):1458-65.

2. BLACK TLP, CARDOSO MD. Coinfecção TB/HIV em adolescentes residentes em Pernambuco, notificados no período de 2001 a 2016. Braz. J. Hea. Rev. 2019;2(1):321-334.

3. BRUCHFELD J, et al. Tuberculosis and HIV Coinfection. Cold Spring HarbPerspect Med. 2015:5: a017871

4. CASTRIGHINI CC, et al. Prevalência e aspectos epidemiológicos da coinfecção HIV/tuberculose. Rev enferm UERJ. 2017; 25:1-6. 
5. CONCEIÇÃO EL. Avaliação de fatores genéticos e imunológicos relacionados à imunopatogênese da tuberculose e co-infecção TB-HIV. Tese (Pós-graduação em Imunologia) - Instituto de Ciências da Saúde. Universidade Federal da Bahia, Salvador; 2016. 123p.

6. ESCADA ROS, et al. Mortality in patiens with HIV-1 and tuberculosis co-infection in Rio de Janeiro- associeated factors and causes of death.BMC Infect Dis. 2017; 7(1): 373.

7. FERREIRA DP, et al. Prevalência da Coinfecção Hiv/Tb em Pacientes de um Hospital de Referência na Cidade do Rio De Janeiro. J. Rev Fundam. Care. Online.2019; 11: 358-362

8. GALVÃO JMV, et al. Co-infection in hiv/aids-bearing people from a specialized care service of the interior region of Maranhão state. Rev Fun Care Online. 2019;11(4):1103-1110.

9. GONÇALVES HF. Perfil clínico e epidemiológico e prevalência de coinfecção de tuberculose-hiv no estado de Pernambuco, Brasil. Dissertação (Graduação) - Centro Acadêmico de Vitória de Santo Antão. Universidade Federal de Pernambuco, Pernambuco; 2018, 39p.

10. HINO P, et al. Coinfecção de Tb/HIV em um distrito administrativo do município de São Paulo. Acta paul. enferm. 2012;25(5):755-761.

11. LIMA MD, et al. Mortalidade relacionada à coinfecção tuberculose e HIV/AIDS no Brasil, 2000-2011: padrões epidemiológicos e tendências temporais. Cad. Saúde Pública. 2016;32(10):1-11.

12. LIMA SS, et al. Análise espacial da tuberculose em Belém, estado do Pará, Brasil. Rev Pan-Amaz Saude. 2017;8(2):57-65.

13. MACNEIL A, et al. Global Epidemiology of Tuberculosis and Progress Toward Achieving Global Targets-2017. MMWR Morb Mortal Wkly Rep. 2019;68(11):263-266.

14. MAGNO ES, et al. Fatores associados à coinfecção tuberculose e HIV: o que apontam os dados de notificação do Estado do Amazonas, Brasil, 2001-2012. Cad. Saúde Pública. 2017; 35(5): 1-11.

15. MANN D, et al. Cutaneous tuberculosis and HIV infection at a referral centre in Rio de Janeiro, Brazil. Mem Inst Oswaldo Cruz. 2018;113(9):1-6.

16. MARQUES CC, et al. Casos de tuberculose coinfectados por HIV em um estado do nordeste brasileiro. Rev Enferm Act. 2019; 36:62-76.

17. MCQUAID CF, et al. The potential impact of COVID-19-related disruption on tuberculosis burden. Eur Respir J. 2020 ;56(1):1-9.

18. MIRANDA LO, et al. Aspectos epidemiológicos da coinfecção Tuberculose/HIV no Brasil: revisão integrativa. Rev Pre Infec e Saúde. 2017;3(3): 59-70.

19. OLIVEIRA EH, et al. Aspectos epidemiológicos da coinfecção tuberculose/HIV: análise de uma década em Teresina, Piauí, Brasil. Rev Pre Infec e Saúde. 2020;9(5):1-5.

20. OLIVEIRA HB, et al. Tuberculosis fatality rates in the city of Campinas - São Paulo, Brazil, from 2001 to 2009 . Rev Bras Epidemiol. 2019; 22: 1-11.

21. OLIVEIRA LB, et al. Epidemiological Analysis of Tuberculosis/HIV Coinfection. Cogitare Enferm. 2018;23(1):1-8.

22. OLIVEIRA NF, GONÇALVES MJF. Fatores sociais e ambientais associados à hospitalização de pacientes com tuberculose. Rev. Latino-Am. Enfermagem. 2013; 21(2): 507-514.

23. ORCAU A, et al. Present epidemiology of tuberculosis. Prevention and control programs. Enferm Infecc Microbiol Clin. $2011 ; 29(1): 2-7$.

24. PEREIRA LFB, et al. Tuberculosis/HIV coinfection associated factors regarding the 2001-2011 timeframe. Rev Fun Care Online. 2018;10(4):1026-1031.

25. PRADO TN, et al. Clinical and epidemiological characteristics associated with unfavorable tuberculosis treatment outcomes in TB-HIV co-infected patients in Brazil: a hierarchical polytomous analysis. Braz $\mathrm{J}$ Infect Dis. 2017;21(2):162-170.

26. QUEIROZ CA, et al. Tuberculose associada à AIDS: uma análise da prevalência de coinfecção. Ciência et Praxis. 2018;11(21):65-70.

27. ROSSETTO M, et al, Teixeira LB. Perfil epidemiológico de los casos de tuberculosis con coinfección VIH en Porto Alegre. Rev Bras Enferm. 2019;72(5):1211-1218.

28. ROSSETTO M, et al. Coinfecção tuberculose/HIV/aids em Porto Alegre, RS -invisibilidade e silenciamento dos grupos mais afetados. Rev. Gaúcha Enferm. 2019;40(e20180033):1-9.

29. SANTOS AR, et al. Perfil clínico e epidemiológico da tuberculose no município de Juara, Estado de Mato Grosso. Revista Inspirar movimento \& saúde. 2020;20(1):1-14.

30. SANTOS MLSG. A dimensão epidemiológica da coinfecção TB/HIV. Rev. Latino-Am. Enfermagem. 2009;17(5):8489.

31. SANTOS NM, et al. Perfil clínico e epidemiológico e prevalência da coinfecção tuberculose/HIV em uma regional de saúde no Maranhão. J Bras Pneumol. 2012;38(6):724-732.

32. SILVA BEB, et al. Prevalence of coinfections in women living with human immunodeficiency virus in Northeast Brazil. Rev Soc Bras Med Trop. 2020;53(e20190282):1-6.

33. SILVA WA, et al. Perfil epidemiológico dos casos notificados de tuberculose no Piauí nos anos de 2010 a 2014 . R. Interd. 2017;10(3):31-38.

34. SIQUEIRA-FILHA NT, et al. The economic burden of HIV and TB/HIV co-infection in a middle-income country: a costing analysis alongside a pragmatic clinical trial in Brazil. Sexually Transmitted Infections. 2018;94(6):463-469.

35. SOUSA AG, et al. Contextualização de aspectos sociais da coinfecção TB/HIV no Distrito Federal. Revista Eletrônica Gestão \& Saúde. 2013;4(1):1234-1247.

36. SOUSA FILHO MP, et al. Pacientes vivendo com HIV/AIDS e coinfecção tuberculose: dificuldades associadas à adesão ou ao abandono do tratamento. Rev. Gaúcha Enferm. $2012 ; 33(2): 139-145$.

37. TADOLINI M, et al. On Tuberculosis and COVID-19 co-infection. Eur Respir J.2020;56(1):1-7.

38. TEIXEIRA F, et al. Human Immunodeficiency Virus and Tuberculosis Coinfection in a Tertiary Hospital in Southern Brazil: Clinical Profile and Outcomes. Microbiol Insights. 2018; 11:1-8.

39. VENDRAMINI SHF, et al. Análise espacial da co-infecção tuberculose/HIV: relação com níveis socioeconômicos em município do sudeste do Brasil. Rev. Soc. Bras. Med. Trop. 2010;43(5):536-541. 